\section{Exosome brainstorm}

\section{By Lev Osherovich, Senior Writer}

A Harvard team has shown that monitoring microvesicles called exosomes in serum could be useful for diagnosing glioblastoma and evaluating disease progression. ${ }^{1}$ The work has spawned a new cancer diagnostics company, Exosome Diagnostics Inc., which has started clinical trials of its diagnostic technique for colon cancer, melanoma and glioblastoma multiforme.

Exosomes, also known as oncosomes, are 30-100 nm in diameter. They are enclosed by membranes and contain tumor-associated proteins such as epidermal growth factor receptor variant III (EGFRvIII), which is found in about $50 \%$ of glioblastoma multiforme (GBM) tumors.

Canadian researchers had previously shown that exosomes are secreted by cultured brain tumor cells and spread cancer-driving proteins to bystander cells, ${ }^{2}$ whereas other studies have shown that cultured cell lines derived from aggressive tumors such as certain breast cancers and GBM could shed exosomes into the sur-
"I doubt that this study has gone far enough to show that microvesicles have the potential to induce new tumors."

- Venky Ramakrishna, Celldex Therapeutics Inc.
The team detected "more than 20,000 different messages in the exosome," said Breakefield. That suggested the microvesicles had escaped with a sample of the cancer cell's cytoplasm, which contains a multitude of mRNAs that are differentially expressed in tumor cells compared with healthy cells. Indeed, the exosomes contained high levels of 11 miRNAs that are overexpressed in GBM tumors compared with in normal cells.

The findings allowed Skog and Breakefield's team to use the abnormal RNA profile of exosomes as a biomarker for the originating tumor: rather than looking for tumor proteins in serum, the team looked for RNA. Detecting small amounts of RNA is much easier than directly detecting the exosome-borne tumor proteins, thanks to techniques such as reverse transcription PCR (RT-PCR).

"We can pick up nucleic acids from the tumor and detect specific mutations" in the exosome-borne RNA that match those of the tumor, said Skog.

To test the method, the team performed RT-PCR on brain tissue and serum exosome samples from 30 GBM patients, looking for EGFRvIII mRNA. Among the biopsy samples, 14 patients came up positive. The exosome samples correctly identified the status of 5 of these patients, with 9 false negatives and 2 false positives. EGFRvIII mRNA was not detected in the serum of 30 healthy controls.

The team obtained similar results with a GBM-linked miRNA, miRNA-21.

Skog believes that searching for tumorderived RNA in the serum could be a sensitive and noninvasive way to screen for early signs of rounding medium, where they fuse with and transform nondividing cells. ${ }^{3}$

Until now, it was not known whether tumor-derived exosomes were present in cancer patients or whether they were a cell culture artifact.

The Harvard study dispels some of these doubts by proving the existence of exosomes in GBM patients. The challenge now is to refine the detection method to create a useful diagnostic and to show that exosomes indeed contribute to the transformation of healthy human cells in vivo.

\section{Sensitivity training}

In the study published in Nature Cell Biology, a team led by Xandra Breakefield, professor of neurology at Harvard Medical School and professor of genetics at Massachusetts General Hospital (MGH), developed a way to purify and analyze the contents of exosomes shed by primary cultures of GBM cells.

Johan Skog, an instructor of neurology at MGH and lead author of the study, said that although exosomes have been studied for more than 30 years, the breakthrough in the new study was the discovery that the structures contain not only proteins, but also mRNA and microRNA.

"This is the first time we can detect tumor mutations in nucleic acids from exosomes," said Skog, who is also director of genetics research at Exosome Diagnostics.

The group used a 44,000-gene microarray to analyze the mRNA content of both the exosomes and the originating tumor.
GBM. However, the poor performance of the approach in distinguishing between EGFRvIII-negative and EGFRvIII-positive tumors means that the preparation and analysis of exosome RNA will need to be optimized.

Skog told SciBX that decreasing the size of the RT-PCR-amplified sequence might improve sensitivity because PCR yields for sequences shorter than the entire EGFRvIII gene tend to be higher, thus lowering the false-negative rate.

In addition, Skog said working with fresher serum and tissue samples from live patients, rather than the frozen samples used in the study, has improved the detection rate.

Exosome Diagnostics, which has licensed patents filed by MGH based on Skog and Breakefield's work, says it has already improved on the procedure.

According to Chairman and CEO James McCullough, over the last year the company has optimized the microvesicle preparation procedure and has eliminated the false-negative problem.

"The sensitivity of the assay has increased" compared with the results in the Nature Cell Biology study, he said. "We are confident of the process."

\section{Diagnostic prospects}

Researchers told SciBX that the Nature Cell Biology study could aid the early detection of brain tumors, which are typically only diagnosed at late stages. 


\section{TARGETS \& MECHANISMS}

"There's a lot of work going on about the detection of nucleic acids in the serum" to diagnose early-stage tumors, said Joe Gray, director of the life sciences division of Lawrence Berkeley National Laboratory. "The microvesicle concept allows us to increase the sensitivity of detection."

Although GBM exosomes have been seen before, the new study "has taken the observation into the clinical realm, where they've been able to detect some crucial mRNA species by PCR," said Janusz Rak, associate professor of pediatrics at McGill University. Rak authored the earlier exosome study that was also published in Nature Cell Biology.

Rak said that the first application of the method could be to stratify GBM patients. Compared with a biopsy, a serum test "could be a better way to detect whether a patient is EGFRvIII-positive or -negative," he said.

Knowing a patient's EGFRvIII status could shape therapeutic decisions. For EGFRvIII-positive tumors, small molecule inhibitors such as Tarceva erlotinib from OSI Pharmaceuticals Inc., Genentech Inc. and Roche are reasonable treatment options, according to Rak.

Tarceva received Orphan Drug designation for GBM in 2003 and is in Phase I/II trials for the indication. The drug is marketed for non-small cell lung cancer (NSCLC) and pancreatic cancer.

Rak told SciBX that the exosome sampling and analysis technique appears to soon be ready for clinical trials, adding that its usefulness as a marker will need to be validated in a larger group of patients.

Meanwhile, he recommended testing whether differences in the RNA content of exosomes between patients provide clues about disease progression and prognosis.

The RNA content of exosomes "is not a random pinching of cellular material, but rather it's a selective process," said Rak. "We need to know what it means for a particular cancer to have a particular set of microvesicle-associated transcripts."

One company, Celldex Therapeutics Inc., is developing a therapeutic vaccine for GBM that specifically targets EGFRvIII and could potentially benefit from exosome-based tumor profiling. The company's CDX-110 peptide vaccine is in Phase III trials for GBM and is partnered with Pfizer Inc.

Venky Ramakrishna, director of immunology at Celldex, said Skog and Breakefield's study was an advance in defining the epigenetic mechanisms of GBM, but he questioned whether exosomes are a cause or consequence of pathology and even whether they originate from tumors in vivo.

"I doubt that this study has gone far enough to show that microvesicles have the potential to induce new tumors," said Ramakrishna. "It still needs to be demonstrated that in situ you can detect microvesicles."

As an alternative hypothesis, Ramakrishna suggested that serum exosomes could be "biological response modifiers that are either produced by normal tissue or are associated with a constantly inflammatory environment."

Regardless of the exosomes' origin, Exosome's McCullough said, a serum-based test for cancer markers could help to tailor therapeutic strategies.

The company is focused on extracting nucleic acids from exosomes in blood and urine, with the goal of developing a laboratory service assay to detect cancer-associated mutations in EGFR, $\mathrm{v}$-Ki-ras2 Kirsten rat sarcoma viral oncogene homolog (K-RAS) and $\mathrm{V}$-raf murine sarcoma viral oncogene homolog B1 (B-RAF). The latter two oncogenes are mutated in aggressive lung and colon cancers, and melanoma, respectively.

McCullough said Exosome has started clinical trials of its diagnostic technique for colon cancer, melanoma and GBM.

Going forward, Skog plans to characterize the RNA content of exosomes produced by other types of tumors.

Meanwhile, Breakefield wants to follow up on the effect of tumorderived RNA on normal cells that absorb exosomes from the serum. The team found evidence that exosome mRNAs encoding proangiogenic factors are translated and stimulate angiogenesis in cell culture.

"People never realized before that cancer cells can send out a protected packet of genetic information," said Breakefield. She suspects that cancer cells send out exosomes "to mold the environment to facilitate tumor growth."

Osherovich, L. SciBX 1(44); doi:10.1038/scibx.2008.1062

Published online Dec. 11, 2008

\section{REFERENCES}

1. Skog, J. et al. Nat. Cell Biol.; published online Nov. 16, 2008; doi:10.1038/ncb1800

Contact: Xandra Breakefield, Massachusetts General Hospital, Charlestown, Mass.

e-mail: breakefield@hms.harvard.edu

Contact: Johan Skog, same affiliation as above e-mail: Skog.Johan@mgh.harvard.edu

2. Al-Nedawi, K. et al. Nat. Cell Biol. 10, 619-624 (2008)

3. Osherovich, L. SciBX 1(16), 1-4; May 15, 2008

COMPANIES AND INSTITUTIONS MENTIONED

Celldex Therapeutics Inc. (NASDAQ:CLDX), Phillipsburg, N.J.

Exosome Diagnostics Inc., New York, N.Y.

Genentech Inc. (NYSE:DNA), South San Francisco, Calif.

Harvard Medical School, Boston, Mass.

Lawrence Berkeley National Laboratory, Berkeley, Calif.

Massachusetts General Hospital, Boston, Mass.

McGill University, Montreal, Quebec, Canada

OSI Pharmaceuticals Inc. (NASDAQ:OSIP), Melville, N.Y.

Pfizer Inc. (NYSE:PFE), New York, N.Y.

Roche (SWX:ROG), Basel, Switzerland 